\title{
Movimientos sociales, espacio público y ciudadanía: Los caminos de la utopía
}

Social movements, public space, and citizenship: The paths of utopia

Mouvements sociaux, espace public et citoyenneté : les chemins de l'utopie

\section{Benjamín Tejerina}

\section{OpenEdition}

\section{Journals}

Edición electrónica

URL: http://journals.openedition.org/rccs/982

DOI: $10.4000 /$ rccs.982

ISSN: 2182-7435

Editor

Centro de Estudos Sociais da Universidade de Coimbra

\section{Edición impresa}

Fecha de publicación: 1 octubre 2005

Paginación: 67-97

ISSN: 0254-1106

Referencia electrónica

Benjamín Tejerina, « Movimientos sociales, espacio público y ciudadanía: Los caminos de la utopía », Revista Crítica de Ciências Sociais [En línea], 72 | 2005, Publicado el 01 octubre 2012, consultado el 10 diciembre 2020. URL : http://journals.openedition.org/rccs/982 ; DOI : https://doi.org/10.4000/rccs 982 


\section{Movimientos sociales, espacio público y ciudadanía: Los caminos de la utopía}

Ella está en el horizonte - dice Fernando Birr -. Me acerco dos pasos, ella se aleja dos pasos. Camino diez pasos y el horizonte se corre diez pasos más allá. Por mucho que yo camine, nunca la alcanzaré. ¿Para qué sirve la utopía? Para eso sirve: para caminar.

EduARDo GaLEANO, "Las palabras andantes”

El objeto de este artículo son las relaciones que se establecen entre los movimientos sociales y los procesos de construcción de la ciudadanía mediante la reapropiación y resignificación tanto física como simbólica del espacio público. Desde los estudios de la acción colectiva el espacio público se ha comprendido tradicionalmente como el escenario en el que tienen lugar las disputas por la legitimidad de las demandas colectivas. Pero lo que sucede en el espacio público tiene una conexión directa con los espacios de la privacidad, con los intereses privados y con la agregación de estos intereses en redes de socialidad que conectan diversas individualidades. Una especie de privacidad compartida que se hará visible cuando la movilización política ocupe el espacio público. La cristalización de las demandas que se formulan en la privacidad compartida produce la modificación del ámbito de derechos y responsabilidades de ciudadanía que cuestiona y pone en crisis los límites de la política institucional (izada).

\section{Introducción}

El resultado de los análisis centrados en los conflictos que históricamente han ocupado el espacio público ha sido triple: en primer lugar, la invisibilización de los mecanismos sociales de construcción de la protesta que tienen lugar fuera del espacio público, pero que dotan de contenido a la movilización política cuando ésta decide ocupar las calles y las plazas; en segundo lugar, el ocultamiento - sin duda no intencional - de otras formas de ciudadanía vicarias de la ciudadanía política que ha hejemonizado el escenario público en las sociedades industriales, aunque esta afirmación bien podría extenderse a otros modelos de sociedad; y, en tercer lugar, el espacio público es un espacio considerado como socialmente instituido, espacio de encuen- 
tro y desencuentro entre autoridades y demandantes, cuyas condiciones constituyentes no se problematizan. Lo que pretendo en el corto espacio de este artículo es reducir la invisibilidad, el ocultamiento y la aproblematicidad con los que, en ocasiones, se ha tratado de explicar los procesos de constitución de la ciudadanía en las sociedades modernas. Para ello me ocuparé, en primer lugar, del concepto tradicional de ciudadanía y la constitución del espacio público; en segundo lugar, de los procesos de movilización y politización que forma el espacio público a partir de la década de los años 70; en tercer lugar, de las condiciones de producción de ciudadanías vicarias que requieren de un proceso de elaboración identitario previo a su aparición en el espacio público y su desarrollo como realidad socialmente conflictiva; en cuarto lugar, me detendré en algunas de las redefiniciones de la ciudadanía que han alcanzado un alto grado de visibilidad política.

El debate sobre las relaciones entre la esfera pública, la movilización social y la ciudadanía es relevante porque plantea tres problemas de alcance teórico: en primer lugar, los procesos históricos de definición y redefinición de los contenidos sociales de la ciudadanía; en segundo lugar, la relación entre visibilidad e invisibilidad de la movilización, entre su construcción en redes sumergidas y su expresión/reproducción en la esfera pública; y en tercer lugar, la posibilidad de repensar la dicotomía espacio privado versus espacio público, lo personal versus lo político, tanto en términos sociológicos como históricos.

\section{De siervo a caballero: la incierta marcha de la ciudadanía hacia la modernidad}

Para Alfred Marshall (1925) existe una igualdad humana básica asociada al concepto de la pertenencia plena a una comunidad, es decir, a la ciudadanía. Esta semejanza cualitativa es compatible, en principio, con todo tipo de desigualdades que existen en la sociedad y, en primer lugar, con las desigualdades económicas. Entendía la ciudadanía como un valor "que el artesano cualificado aprendía a apreciar en el curso de su conversión en caballero". Se trata de un estilo de vida que se cultiva dentro de la persona, que no se le presenta desde fuera. Con el paso del tiempo este núcleo central de igualdad humana básica de pertenencia a una comunidad se ha enriquecido con la formulación de nuevos derechos.

T. H. Marshall ha dividido la ciudadanía en tres partes o elementos: civil, política y social.

El elemento civil se compone de los derechos necesarios para la libertad individual: libertad de la persona, de expresión, de pensamiento y religión, derecho a la propiedad y a establecer contratos válidos y derecho a la justicia. [...] Por elemento político 
entiendo el derecho a participar en el ejercicio del poder político como miembro de un cuerpo investido de autoridad política, o como elector de sus miembros. [...] El elemento social abarca todo el espectro, desde el derecho a la seguridad y a un mínimo bienestar económico al de compartir plenamente la herencia social y vivir la vida de un ser civilizado conforme a estándares predominantes en la sociedad. (Marshall, 1998: 22-23)

En un determinado momento de la historia tanto los derechos políticos de una persona como los civiles e, incluso, los sociales formaban una amalgama y dependían del estatus que, a su vez, determinaba la justicia que disfrutaba, dónde podía encontrarla y las posibilidades de participar en los asuntos de la comunidad a la que pertenecía. Como acertadamente señala Marshall

[S]in embargo, no se trataba de un estatus de ciudadanía en el sentido moderno. El estatus de la sociedad feudal era el sello de clase y la medida de la desigualdad. No existía un conjunto uniforme de derechos y obligaciones para todos - nobles y plebeyos, libres y siervos -, en virtud de su pertenencia a la sociedad. En ese sentido, no se disponía de un principio de igualdad de los ciudadanos para contrarrestar el principio de desigualdad de las clases. Por otra parte, en las ciudades medievales se podían encontrar ejemplos de ciudadanía auténtica igual pero mientras que sus deberes y obligaciones característicos eran estrictamente locales, la ciudadanía cuya historia quisiera trazar aquí es nacional por definición. (Marshall, 1998: 24)

El análisis de Marshall continúa señalando que en un determinado momento los tres elementos de la ciudadanía se separaron, rompieron su relación, hasta el punto que "tan completo fue el divorcio que, sin violentar demasiado la precisión histórica, podemos asignar el periodo formativo de cada uno a un siglo distinto - los derechos civiles, al siglo XVIII; los políticos, al XIX; y los sociales al XX” (Marshall, 1998: 25-26). Más allá de la certeza de esta generalización empírico-histórica, lo que me interesa retener es lo que queda oculto: cómo la ciudadanía cívica llevó a cabo un proceso de movilización que terminó ampliándose hacia las ciudadanías política y social.

Un logro del movimiento obrero organizado y de los conflictos sindicales en los que se implicó ha sido posibilitar la transformación de derechos civiles individuales en colectivos, creando una especie de "ciudadanía industrial secundaria" que utilizaba los derechos civiles colectivos tanto para negociar su aplicación como para consolidar o ampliar los derechos civiles fundamentales mismos. En el desarrollo de la ciudadanía política encontramos una lógica social expansionista que coloniza progresivamente 
las otras esferas de la ciudadanía: la civil y la social. La creciente incorporación de nuevas áreas a los derechos civiles y sociales, incluida su redefinición, ha venido de la mano de la confrontación en la esfera pública de las fuerzas a favor de su ampliación a nuevos sectores sociales (ante la agitación del fantasma de la revolución) y los partidarios de su contención o reducción (bajo la escenificación del peligro de la ingobernabilidad y la amenaza a la privacidad).

De los escritos de Marshall cabe diferenciar dos versiones de la ciudadanía: la formal (derechos civiles) y la sustantiva (derechos políticos y sociales). La primera se adquiere mediante la pertenencia a un Estado-nación y, la segunda, consistiría en un conjunto de derechos civiles, políticos y sociales que implican la participación en los asuntos del gobierno. Como señala Rogers Brubaker:

Lo constitutivo de la ciudadanía - el conjunto de derechos o el modelo de participación - no se encuentra necesariamente vinculado a la pertenencia formal a un Estado. La ciudadanía formal no es condición suficiente ni necesaria para la ciudadanía sustantiva $[. .$.$] como se aprecia claramente en el hecho de que perteneciendo$ formalmente a un Estado se puede estar excluido (legalmente o de hecho) de ciertos derechos políticos, civiles o sociales, o de la participación efectiva en asuntos de gobierno relativos a los más variados aspectos de la vida social [...] y ello es así aunque no nos parezca evidente, pues si la ciudadanía formal puede resultar necesaria para ciertos componentes de la ciudadanía sustantiva (por ejemplo, votar en las elecciones generales), hay otros componentes [...] que no dependen de la pertenencia formal a un Estado. Los derechos sociales, por ejemplo, benefician tanto a los ciudadanos como a los residentes legales no nacionalizados, en condiciones prácticamente idénticas, así como la participación en la gestión de las asociaciones, los partidos políticos, los sindicatos, los consejos de fábrica y otras instituciones. (Brubaker, 1992: 36)

Los derechos de ciudadanía no han tenido una aplicación lineal, puesto que amplios colectivos han sido sistemáticamente excluidos de su alcance. En algunos casos, los derechos políticos y sociales no acompañan al reconocimiento de los derechos civiles (formales) y, en otros casos, los derechos civiles encuentran trabas jurídicas limitadoras de su aplicación universal.

Un primer ejemplo. La implantación del conjunto de derechos civiles, políticos y sociales, entre las mujeres ha encontrado siempre fuertes resistencias, lo que motivó la aparición del movimiento sufragista en Gran Bretaña y EE.UU. a finales del siglo XIX, y la reformulación de un nuevo discurso feminista a partir de la década de los años 60 del siglo XX. Como ha señalado Tom Bottomore: "las mujeres disfrutaron mucho más tarde de 
los derechos civiles - por ejemplo, la propiedad -, y existen ejemplos, como el de Gran Bretaña, donde los impuestos sobre la renta de las casadas no se consideraron independientemente, sino como una extensión de los ingresos del marido, hasta 1990. Durante el siglo XX, en la mayoría de los países, los derechos políticos llegaron también mucho más tarde para las mujeres - en algunos casos después de 1945 -, que aún forman una pequeña minoría en las asambleas legislativas y en los altos cargos de la administración estatal, si bien participan de forma activa y en puestos sobresalientes en los movimientos sociales. En el terreno de los derechos sociales se ha discriminado a la mujer - y aún se la discrimina en muchos países - en lo relativo a la remuneración, perspectivas de promoción y prestigio de sus ocupaciones, mientras que la provisión social de las áreas que le afectan directamente, como guarderías diurnas, permisos de maternidad y planificación familiar, se ha expandido con una rapidez muy inferior a la de otros servicios" (Bottomore, 1998: 103-104).

Un segundo ejemplo. La limitación en la extensión de los derechos de ciudadanía a colectivos étnicamente diversos o con pautas etnoculturalmente diferenciadas de las preexistentes en las sociedades a las que han emigrado con posterioridad a la segunda Guerra Mundial. La inmigración, y la diversidad étnica, lingüística, religiosa o cultural, que incorpora a la sociedad de recepción, plantea problemas relativos "tanto a la ciudadanía formal como a la ciudadanía sustantiva, y las políticas que afectan a la primera varían considerablemente de un país a otro - por ejemplo, entre Alemania, Francia y Gran Bretaña -, aunque la tendencia general de las décadas pasadas ha consistido en restringir la inmigración y el acceso a la ciudadanía" (Bottomore, 1998: 105).

Un tercer ejemplo. Al menos desde la década de 1960, y en algunos casos desde mucho antes, se vienen manifestando de manera constante tendencias a la disgregación dentro de estados-nación ya constituidos por parte de grupos con una personalidad propia; personalidad que se articula en la existencia sobre un determinado territorio de hechos diferenciales tales como una historia, una lengua, una cultura o ciertas costumbres características. Más allá del proceso de construcción de la diversidad cultural, la movilización por el reconocimiento de una nacionalidad distinta y separada dentro del Estado-nación plantea problemas relativos a la ciudadanía "cuando existen grupos - por ejemplo, en la provincia de Quebec (Canadá), en el País Vasco (España), en Irlanda del Norte y, cada vez más, en la Europa del este después de la caída de los regímenes del socialismo estatal - que crean movimientos reivindicativos [...] en la forma de independencia total, o al menos de una amplia autonomía regional" (Botto- 
more, 1998: 106). Más adelante volveremos sobre los procesos de ampliación de la ciudadanía en la actualidad, en este momento me interesa resaltar el carácter social e históricamente construido de esta concepción de la ciudadanía.

Es innegable el carácter parcial de la consecución de la ciudadanía civil, de la que han sido excluidos colectivos importantes, y ello se debe a la influencia de la clase y de los conflictos de clase de los últimos dos siglos.

Fueron los burgueses quienes, oponiéndose a la aristocracia feudal, conquistaron los derechos civiles, y hasta cierto punto los políticos, primero en las ciudades medievales, y luego, a escala nacional, en las primeras fases de desarrollo del capitalismo industrial. La lucha decimonónica por extender los derechos políticos corrió a cargo de los movimientos obreros, en las revoluciones de 1848, el movimiento cartista y las posteriores campañas por el sufragio universal que ocuparon un lugar preeminente en las actividades de los partidos socialistas que en ese momento crecían con rapidez en toda Europa. Las luchas se prolongaron durante el siglo XX, incluyendo ya los derechos sociales, dirigidas sobre todo por los sindicatos y los partidos socialistas, y formaron parte de un movimiento más general por la implantación del socialismo. El Estado del bienestar posterior a la guerra en Europa occidental, resultado, antes que nada, de estas acciones de clase, mantuvo un cierto equilibrio desde finales de los años cuarenta hasta comienzos de los setenta gracias a esa fórmula de "capitalismo del bienestar" y "economía mixta" que Schumpeter calificó de posible "punto medio" en la marcha hacia el socialismo, y que científicos sociales posteriores han llamado neocapitalismo, capitalismo organizado o corporatismo (Panitch). En este sistema de Estado intervencionista han sido fundamentales los acuerdos que negociaron el gran capital y las organizaciones obreras para alcanzar un "compromiso de clase".

(Bottomore, 1998: 111-112)

Así se explica que los derechos reconocidos lleven la impronta de las categorías hombre, trabajador, nacional/nativo, y alguna más que podríamos añadir, quedando fuera de las prioridades aquellos intereses e identidades no coincidentes con las anteriores: mujer, no asalariado, inmigrante, perteneciente a otro grupo étnico, etc. Durante décadas la lógica social dominante en el espacio público ha sido la lógica de clase y la vida política ha girado en torno al ámbito de acción definido por el Estado-nación. Las demás lógicas sociales se subordinaban al monopolio que el Estado detentaba como productor casi exclusivo de la concepción de la ciudadanía en la sociedad industrial moderna. La crisis actual del Estado-nación ha puesto de manifiesto el predominio de esta lógica en la que lo político, lo público y lo social se entronizan de manera indiferenciada como centro sagrado de la moder- 
nidad. Únicamente con la crisis del Estado de clase, Estado del bienestar, es posible la emergencia al espacio público de otras lógicas sociales que funcionan en los márgenes o al margen de la identidad de clase.

\section{La politización de la política y la socialización de la ciudadanía}

La existencia de un espacio público político definido desde el Estado ha terminado identificando Estado, nación y sociedad, así como la incorporación y pertenencia individual a ella a través de los derechos y obligaciones de la ciudadanía. François Dubet lo ha plasmado en pocas palabras:

El ciudadano es siempre miembro de una comunidad, un grupo, una nación a los que limitan las fronteras de la ciudadanía. La Atenas democrática era también aristocrática. Durante mucho tiempo, únicamente los hombres ricos o propietarios, luego los hombres adultos y más adelante las mujeres fueron ciudadanos. En todos los casos, abarcando un mayor o menor número de individuos, la ciudadanía se consideró ante todo como la expresión de una nación. Una nación de ciudadanos evidentemente, pero una nación definida por sus especificidades, su idioma, su cultura, su historia y, sobre todo, por su deseo de ser una nación. En ese sentido, fuera uno ciudadano de Venecia, Florencia, Francia o Estados Unidos, la ciudadanía se basa en un vínculo de fidelidad a la nación, y ya no sólo en una fidelidad directa y personal al soberano como ocurría en la sociedad feudal.

Consideradas desde esa óptica, las democracias han sido nacionales, y los ciudadanos han sido ante todo patriotas. (Dubet, 2003: 220)

Sólo la política puede poner en peligro la política. En las últimas décadas del siglo XX, la crisis del Estado-nación, la multicrisis de una forma de articular la política y los conflictos de intereses que se había convertido en "demasiado pequeño para manejar los grandes problemas y demasiado grande para resolver los problemas cercanos y cotidianos", se convierte en una crisis de la constitución (poética) del orden social. La crisis del Estado se acelera, paradójicamente, como consecuencia del apaciguamiento de la "lucha de clases". Pero la crisis de la política hace visibles los mecanismos de producción de la política, y los procesos mediante los que un asunto, anteriormente privado, deviene público-político, se transforma en cuestión de discusión pública y de confrontación con los agentes que gestionan la res publica.

Claus Offe ha sabido ver este proceso de construcción social de una esfera pública politizada con la crisis del estado de bienestar y el cuestionamiento del monopolio que sobre el escenario político nacional ejercía el conflicto de clases y los intentos de apaciguarlo. Lo que Claus Offe definió como el viejo paradigma consistía en un consenso extremadamente amplio sobre el 
Estado de bienestar liberal democrático como orden social, económico y político adoptado con posterioridad a la Segunda Guerra Mundial compuesto por tres elementos.

En primer lugar, [...] se institucionalizaron las decisiones acerca de las inversiones como terreno de actuación de los propietarios y gerentes de empresa operando en mercados libres según criterios de rentabilidad. [...] En segundo lugar, se complemento el capitalismo como máquina del crecimiento con la organización de los trabajadores como máquina de la distribución y de seguridad social. Sólo sobre la base de un empeño preferente por el crecimiento y las ganancias reales, se explica tanto la disposición de los trabajadores organizados a dejar de lado proyectos de transformación social de mayor envergadura a cambio de un status firmemente consolidado en el proceso de la distribución de las ganancias, como la disposición por parte de los inversores a garantizar tal status a los trabajadores organizados. [...] En tercer lugar, el elemento más importante del esquema constitucional del período de la posguerra [...] era una forma de democracia política de tipo representativo y mediatizada por competencia entre partidos. (Offe, 1988: 170-171)

La progresiva estabilidad de la que este amplio consenso va a ir gozando en las sociedades capitalistas europeas generará una visión dicotómica de la sociedad moderna: la institucionalización de una esfera pública donde se discuten las cuestiones colectivas entre agentes cualificados para ello y la segregación de una segunda esfera donde se sitúan las cuestiones privatizadas. El supuesto sociológico implícito subyacente anunciaba que "el estilo de vida centrado en la familia, el trabajo y el consumo, absorbería las aspiraciones y energías de la mayor parte de la población, con lo que la participación en la política y en los conflictos políticos tendría en la vida de la gran mayoría de los ciudadanos un significado solamente marginal" (Offe, 1988: 171).

Este modelo de ciudadanía social planteaba tres vías de acceso: la nacionalidad, el empleo y la masculinidad. "Si consideramos la ciudadanía como el sistema central de inclusión, cohesión y reconocimiento (y autorreconocimiento) en una comunidad política a cambio de la posesión de una serie de titularidades y el cumplimiento de un sistema codificado de obligaciones", podemos decir que "amplió el conjunto de bienes públicos y libertades positivas [...], pero en muy poco amplió las vías y condiciones de acceso al disfrute pleno de esos derechos. El carácter masculino, laboral-industrial y nacional de ese modelo de ciudadanía dejaba como grupos, además de explotados, dominados, oprimidos y sin reconocimiento a todos aquellos grupos laborales que no podían representarse en el imaginario social dominante y normalizado de ciudadano occidental" (Alonso, 2004: 5). 
Lo que me interesa señalar es que el consenso del bienestar excluía otras ciudadanías vicarias, fragmentadas o dependientes, relegando a un lugar de invisibilidad "a todos aquellos grupos que aunque trabajando, su actividad no era reconocida ni cultural, ni social, ni legalmente como formadora de propiedad social y conformadora de identidad, sino como ocupación complementaria, coyuntural, transitoria, oportunista y hasta en el peor de los casos parasitaria o desleal" (Alonso, 2004: 6).

La emergencia de nuevas o renovadas ciudadanías vicarias anuncia la crisis de un modelo que va agotando las posibilidades de adaptación a los procesos de transformación de una ciudadanía definida exclusivamente en torno a las categorías hombre, trabajo, nación, público. Estamos ante una ampliación del ámbito de la ciudadanía, de la colonización por su parte de nuevos territorios anteriormente excluidos de su consideración. La ciudadanía, como veremos, transita desde la esfera privada a la pública cuestionando su comprensión dicotómica. Frente a la teoría política liberal que diferencia entre acciones privadas y acciones públicas (las propiamente políticas),

[S]e sitúan los nuevos movimientos sociales en una tercera categoría intermedia. Reivindican para sí mismos un tipo de contenidos que no son ni "privados" (en el sentido de que otros no se sientan legítimamente afectados), ni “públicos” (en el sentido de que se les reconozca como objeto legítimo de las instituciones y actores políticos oficiales), sino que son los resultados y los efectos colaterales colectivamente "relevantes" de actuaciones privadas o político-institucionales de las que, sin embargo, no pueden hacerse responsables ni pedir cuentas por medios institucionales o legales disponibles a sus actores. El campo de acción de los nuevos movimientos sociales es un espacio de política no institucional. (Offe, 1988: 174)

El espacio de "política no institucional" me parece interesante por las paradojas que encierra. En primer lugar, es un espacio construido en torno a "intereses privados" que intentan acudir a la llamada de la redistribución, pero sin formar parte de quien redistribuye los bienes públicos: "las instituciones". En segundo lugar, es un espacio construido en torno a objetivos con la pretensión de que sean asumidos por la colectividad, es decir, se plantean como un proceso de reconocimiento colectivo de una identidad que busca legitimidad social, lo que implica algún grado de, primero, institucionalización y, segundo, participación. No olvidemos que estos intereses colectivos emergen de la esfera privada. En tercer lugar, es un espacio construido por acciones que siendo privadas se han de institucionalizar (en algún grado) para poder ser tenidas en cuenta, pero que por su carácter privatista no son susceptibles de apropiación colectiva sino de reasignación 
privada. Lo que, en mi opinión, tiene de relevante la formulación de Claus Offe es que pone de manifiesto la necesidad de introducir en el debate los procesos no políticos de constitución de una esfera política diferenciada, en tanto que condiciones sociales de posibilidad o pre-políticas, de cómo lo no político deviene político; es decir, las operaciones sociales por las que se acuerda lo que es y lo que no es político, lo que es y no es privado.

En los apartados siguientes me detendré en analizar 1) la ciudadanía como un espacio construido a partir de la apropiación colectiva de algún o algunos aspectos considerados previamente privados, lo que implica una doble lógica social de reconocimiento y redistribución; 2) la centralidad del reconocimiento (identidad) en el proceso de constitución de las ciudadanías vicarias; 3) la redefinición del espacio público y las reformulaciones de la ciudadanía en las sociedades de conocimiento.

\section{Los movimientos sociales como procesos de movilización de ciudadanías: las palabras andantes}

Ningún enfoque sobre los movimientos sociales es tan útil como la teoría de la movilización de recursos si se pretende dar cuenta de la influencia de la asociación de intereses privados para entrar a formar parte de la redistribución de recursos y recompensas que tiene lugar en la sociedad. La cantidad y capacidad de gestión de recursos e incentivos disponibles, así como las oportunidades políticas que el contexto abre a la movilización permiten dar cuenta de la influencia y transformación sociales que produce un movimiento social. Sin embargo, en el ámbito del reconocimiento, los límites autoimpuestos por la teoría de la elección racional y las limitaciones que encuentran los enfoques de la movilización de recursos han sido apuntados por numerosos autores. ${ }^{1}$ Por ejemplo, Myra Ferre ha señalado que "debido al individualismo radical de esta teoría, se hacen muy problemáticos los aspectos relacionados con la búsqueda de una comunidad y el valor motivador de los bienes colectivos. $\mathrm{Al}$ ser un modelo unidimensional de la conducta 'racional' (estratégicamente instrumental), las formas de conducta no instrumental no sólo no pueden ser tratadas, sino que otros sistemas de valores y formas de conocimiento son sistemáticamente excluidos de consideración" (Ferre, 1994: 175).

En alguna de sus versiones, la teoría de la elección racional considera la acción colectiva como un grupo de individuos egoístas que se reúnen para

\footnotetext{
${ }^{1}$ Las reflexiones sobre la crítica de Alessando Pizzorno a la teoría de la movilización de recursos se encuentran ampliamente desarrolladas en Benjamin Tejerina (1998). Para un ejemplo empírico sobre los límites de la teoría de la movilización de recursos puede consultarse Tejerina et al., 1995 .
} 
alcanzar sus objetivos. En este proceso "las relaciones comunitarias y de dominación anteriores al surgimiento de los movimientos no parecen ser relevantes, y se presta poca atención a los activos procesos cognitivos a través de los cuales las personas se perciben a sí mismas como miembros de los grupos y reafirman estas identidades con sus decisiones" (Ferre, 1994: 176).

Una crítica más radical es la elaborada por Alessandro Pizzorno. Para este autor, el análisis de la participación en la acción colectiva que se realiza desde las teorías utilitaristas presupone unas condiciones de información perfecta y una situación en que

La incertidumbre del cálculo individual es superada (parcialmente) por la seguridad de que el mercado social en el que los beneficios sociales (prestigio, honor, afecto; el "reconocimiento", en una palabra) pueden ser consumidos permanecerá inalterado. Pero aquí entramos en el campo de la formación de la identidad colectiva. Durante el proceso de su formación, el individuo no puede comparar sus costes actuales con los beneficios futuros porque no posee todavía el criterio (la identidad) con que evaluarlos. Su único objetivo (en el caso puro) es entonces el de formar su propia identidad, esto es, el de asegurar un mercado que acepte (reconozca) su propia moneda. Si alguno trata de "hacer el viaje gratis", obteniendo los beneficios derivados de la acción colectiva sin pagar los costes de la participación, acaba simplemente por quedarse sin reconocimiento. (Pizzorno, 1994: 136)

La identidad colectiva y su reconocimiento resultan fundamentales en la competencia entre grupos. La competencia entre individuos utiliza distinto tipo de recursos que la competencia entre grupos. Cuando los grupos luchan por alcanzar mayor cantidad de un determinado producto social, lo hacen mediante la utilización de la movilización o la amenaza de determinadas acciones políticas. Lo que la sociedad alcanza a cambio es el consenso social por parte de estos grupos. Por otro lado, algunos grupos pueden reclamar un cambio en las reglas del juego de la competencia, sobre todo en el caso de nuevos colectivos o nuevas demandas sociales. Pueden aparecer grupos interesados racionalmente en modificar unas normas que no les benefician o, por decirlo en otros términos, el grupo no se identifica con el mantenimiento de las reglas del sistema.

En la sociedad aparecen con cierta frecuencia grupos que plantean intereses específicos. Pero estos intereses deben ser reconocidos (identificados) y deben movilizarse colectivamente. Como quiera que nos movemos en ámbitos de recursos limitados (económicos) o conflictivos (simbólicos), unos intereses tenderán a verse sobrerrepresentados en la medida que la agregación incrementa el poder de un grupo mientras que otros estarán 
subrepresentados o se verán privados de representación. Este proceso opera sobre un mecanismo de exclusión, ya que las circunstancias tienden a limitar los intereses que pueden ser representados, permitiendo la absorción de la presión de ciertos intereses y rechazando o reduciendo otros. Al mismo tiempo, la organización de la representación introduce una distorsión en los mecanismos de mercado o entre grupos que compiten por recursos escasos. El proceso de representación funciona con un recurso específico que podemos llamar militancia, participación o movilización, y son los representantes (líderes, activistas) los poseedores de dicho recurso. En el momento en que estos intereses se organizan, los representantes tienen que buscar fórmulas para hacer compatibles los objetivos inmediatos con los intereses a largo plazo de sus representados. Llegamos así a la paradoja de que "la máxima utilitarista según la cual cada individuo es el mejor juez de sus propios intereses sólo resulta válida en condiciones de información perfecta. En el mundo real, al contrario, la acción se desarrolla siempre en condiciones de incertidumbre. La representación es un instrumento para reducir la incertidumbre. Un sistema representativo presupone que el mejor juez de los intereses a largo plazo de un individuo es su representante" (Pizzorno, 1994: 140).

Los intereses defendidos por aquellos grupos excluidos tienen que ser reconocidos por los grupos que constituyen el sistema. Ambos tipos de grupos se encuentran en situaciones muy distintas. Mientras que los grupos antiguos encuentran representación para la defensa de intereses definidos y reconocidos, los nuevos grupos luchan por conseguir el ingreso en el sistema y ser reconocidos como representantes de los nuevos intereses a través de un procedimiento distinto, que Pizzorno denomina de "formación de identidades colectivas". En este proceso constitutivo, las acciones desarrolladas por los grupos no están orientadas hacia la maximización del beneficio personal, sino hacia la consolidación de la identidad grupal. En esta situación "tal objetivo no es negociable, se coloca más bien como la premisa de eventuales negociaciones e intercambios futuros. Durante esta fase, cierto tipo de acciones (como los conflictos, la polarización de posiciones, las opciones de coherencia ideológica, la adopción de objetivos no realistas) que parecerían 'irracionales' desde el punto de vista de los beneficios individuales, adquieren, por el contrario, significado si se consideran en la perspectiva de la formación de identidad" (Pizzorno, 1994: 141).

Diferenciando estos dos momentos en el proceso de construcción de la identidad colectiva es posible superar las limitaciones de la teoría de Mancur Olson (1965) sobre la acción colectiva, así como la disputa entre comportamiento patológico y racional, ya que buena parte de las manifestaciones de la 
conducta colectiva en su proceso inicial de génesis responde a una racionalidad de formación de identidad y no tanto al cálculo individual utilitarista. Durante esta fase de formación de la identidad colectiva se intensifica la participación y se incrementa la dedicación a la militancia. Con posterioridad

[U]na vez alcanzado el objetivo del reconocimiento de la identidad, cuando los objetivos subsiguientes pueden conseguirse a través de la negociación, la participación tiende a caer. [...] En realidad, encontramos a menudo una fase intermedia en la que la nueva identidad colectiva se sitúa todavía como antagónica al sistema. En este caso se verificará probablemente una situación de bloqueo polarizado, en la que algunos miembros participan intensamente, mientras que otros desisten, desanimados por la ineficacia a corto plazo de la acción política. La militancia (incentivada por la fuerte necesidad de nueva identidad y por el alto grado de compromiso con ésta) aumentará entonces paralelamente al declive de la participación general. (Pizzorno, 1994: 143)

La única idea que añadiría a las palabras de Alessandro Pizzorno es que también en la segunda etapa, cuando la identidad colectiva está asentada y goza de reconocimiento, una parte de los recursos y de los esfuerzos de la participación deben orientarse a garantizar la legitimidad de los representados ante los oponentes o contendientes. En realidad, el mantenimiento de la identidad que asegure la continuidad de la comunidad reclama buena parte de los recursos disponibles, sobre todo, simbólicos, pero también materiales. Los objetivos de la comunidad son redefinidos cada cierto tiempo, distintas versiones y definiciones de la realidad comunitaria han de ser contrastadas y debatidas, dando lugar a cambios que han de ser gestionados. Lo que me interesa señalar en este momento es que los límites y destino de la comunidad (identidad) son socialmente construidos, por lo que nuevas ciudadanías se formulan y otras se reformulan con el transcurrir del tiempo dependiendo de cambios en la participación y movilización. En esa tarea de adaptación y transformación de la identidad colectiva también han de invertirse una parte de los recursos disponibles, sin quebrar los hilos que la atan a definiciones pasadas, sin romper con la tradición.

Una idea parecida al concepto de identidad colectiva encontramos en la definición de movimiento social de Alain Touraine, para quien se presenta como una combinación de un principio de identidad, un principio de oposición y un principio de totalidad (Touraine, 1978: 108). Pero, sin duda, quien mejor ha sabido plasmar la idea de la identidad colectiva como elemento central en el análisis de los movimientos sociales ha sido Alberto Melucci. A partir de una crítica de la teoría de la movilización de recursos, en el sentido de que conceptos como recursos discrecionales o estructura de 
oportunidades no responden a realidades "objetivas" sino que son interpretados y evaluados por parte de los actores, Melucci llega a la conclusión de que tal teoría supone la existencia de una identidad colectiva (capacidad de definirse a sí mismo y a su ambiente) a partir de la cual el actor es capaz de construir unas expectativas, y compararlas con la realidad y su estructura de oportunidades. Pero, entre los teóricos de la movilización de recursos, esta identidad construida colectivamente se da por supuesta, sin explicitar nunca sus procesos de elaboración y transformación. Para Melucci una identidad colectiva "es una definición interactiva y compartida, producida por varios individuos que interactúan y que hace referencia tanto a las orientaciones como al ámbito de oportunidades y restricciones en el que tiene lugar su acción" (Melucci, 1989: 34).

La identidad colectiva de la que habla Melucci responde a un proceso de construcción social por parte de los individuos o grupos que forman parte de un movimiento social. Como resultado de un continuo proceso de hacerse y rehacerse o, para ser más exactos, definirse y redefinirse, la identidad colectiva está en constante transformación, lo que rompe la idea de la identidad colectiva como algo que permanece inalterado a lo largo del tiempo con el consiguiente peligro de "reificación". Por otro lado, la identidad colectiva como proceso se distancia de aquella concepción que la considera como algo unitario y coherente. En realidad, dentro del ámbito de una identidad colectiva concreta encontramos definiciones diferentes e incluso contradictorias que compiten entre sí, sin negar la existencia de un acuerdo sobre aspectos más generales de dicha identidad colectiva. Esta segunda consideración nos lleva a la reflexión sobre los elementos constitutivos de la identidad colectiva.

Tres tipos de elementos pueden diferenciarse en una identidad colectiva. En primer lugar, implica la presencia de aspectos cognitivos que se refieren a una definición sobre los fines, los medios y el ámbito de la acción colectiva. Este nivel cognitivo está presente en una serie de rituales, prácticas y producciones culturales que en ocasiones muestran una gran coherencia (cuando son ampliamente compartidos por los participantes en la acción colectiva $\mathrm{o}$, incluso, en el conjunto de una determinada sociedad), y en otras circunstancias presenta una amplia variedad de visiones divergentes o conflictivas. En segundo lugar, hace referencia a una red de relaciones entre actores que comunican, influencian, interactúan, negocian entre sí y adoptan decisiones. Según Alberto Melucci este entramado de relaciones puede presentar una gran versatilidad en cuanto a formas de organización, modelos de liderazgo, canales y tecnologías de comunicación. En tercer lugar, requiere un cierto grado de implicación emocional, posibilitando a los activistas sentirse parte 
de un "nosotros". Puesto que las emociones también forman parte de una identidad colectiva, su significación no puede ser enteramente reducida a un cálculo de costes y beneficios, y este aspecto es especialmente relevante en aquellas manifestaciones menos institucionalizadas de la vida social como son los movimientos sociales (Melucci, 1989, 1995 y 1996).

El concepto de identidad colectiva formulado por Alberto Melucci permite entroncar con aquella tradición teórica clásica de la acción colectiva que se fijaba, sobre todo, en la producción cultural de los movimientos sociales. En esta tradición, Melucci ha sabido ver como nadie la dimensión constructivista de la acción colectiva, al tiempo que resalta los desafíos simbólicos que emergen en las redes sumergidas de los movimientos sociales en un largo proceso de elaboración durante los momentos de latencia o inactividad pública (visibilidad). Como intentaré mostrar más adelante, los movimientos sociales se sitúan en un espacio de privacidad compartida que hace posible la conversión de intereses privados en cuestiones de debate en la esfera pública, y permite encontrar una estructura de plausibilidad para vivir la ciudadanía vicaria.

El gran mérito de Melucci ha consistido en señalar el proceso de producción de la identidad colectiva y su centralidad en la dinámica de la acción colectiva, dando respuesta así a la pregunta que otros enfoques teóricos daban por supuesta a la hora de explicar el cómo de la movilización.

\section{El nombre de las cosas. Política y ciudadanía en las sociedades de conocimiento}

En las sociedades de la información tanto la manipulación de la comunicación como su elaboración en términos de conocimiento tienden a sobreponerse a la dimensión material de la existencia. Al menos eso es lo que afirma Manuel Castells (1998) cuando trata de entender el núcleo constitutivo del momento que vivimos en la actualidad. Ronald Inglehart (1991) lo formula en términos de cambio de valores: del materialismo al postmaterialismo. La idea parece clara: en la sociedad postindustrial o de capitalismo avanzado el poder se garantiza mediante la dominación no tanto económica como simbólica. El conflicto central ya no es la disputa por los bienes materiales entre distintas clases sino por la apropiación simbólica del sentido de las cosas: por el nombre de las cosas. ${ }^{2}$

${ }^{2}$ Sobre la función poiética de la política puede consultarse el trabajo excepcional de Iñaki Martínez de Albeniz (2003). 
La tarea de resignificación de la realidad social atribuye a la política un papel relevante. Pero la política no es capaz de garantizar satisfactoriamente la lluvia de demandas que desde la sociedad se lanzan sobre ella. La presencia de los movimientos sociales en un espacio intersticial, de privacidad compartida, entre los deseos individuales y las instituciones públicas es cada vez más necesaria como motor de la innovación democrática. Como ha señalado Melucci: "En lo que se refiere a las formas de acción que conciernen la vida cotidiana y la identidad individual, los movimientos contemporáneos se distancian del modelo tradicional de la organización política y asumen una creciente autonomía de los sistemas políticos. Esos movimientos van a ocupar un espacio intermedio de la vida social, en el cual se entrelazan necesidades individuales e impulsos de innovación política" (Melucci, 1994: 121).

En las sociedades de conocimiento el carácter reflexivo, artificial y construido de la vida social tiene una especial presencia en la forma de experimental el mundo. Un número importante de estas experiencias que tienen lugar en contextos producidos por la acción social, son presentados y representados por los medios de comunicación de masas. El hecho de que una cantidad creciente de la información que recibimos proceda de las tecnologías de la información aplicadas a la comunicación de masas no hace sino multiplicar la reflexividad de la acción social. Estas condiciones, históricas y gnoseológicas, presentes en las sociedades de conocimiento hacen de la acción colectiva un lugar estratégico para observar el conflicto y el cambio social, la producción de nuevos códigos junto a la reinterpretación del pasado, y la producción consciente de nuevos ámbitos de derechos sociales, al tiempo que se redefine la acción política.

Los movimientos sociales no se limitan a recoger el legado histórico, como huellas del pasado que persisten en los fenómenos colectivos contemporáneos, "sino que contribuyen a configurar nuevas pautas de acción colectiva donde coexisten o se combinan los elementos históricos y culturales".

Los movimientos sociales, lejos de aparecer como síntoma ante situaciones de emergencia que tiene una presencia ocasional y esporádica en la vida social - en cierta manera como algo residual del orden social -, son una realidad dotada de cierta permanencia e institucionalización, puesto que a pesar de tener, en ocasiones, una apariencia cíclica sus efectos no son ni esporádicos ni transitorios. Es más que posible que constituyan hoy un componente estable del funcionamiento del sistema (Eder, 1998), que la acción colectiva haya adquirido un estatus autónomo en tanto que formas institucionalizantes separadas de otras con las que solía 
confundirse en el pasado (como la acción política). Melucci lo expresa en los siguientes términos ${ }^{3}$ :

En la era industrial, los conflictos sociales estaban unidos a las luchas por el reconocimiento de los derechos civiles y la ciudadanía. [...] Cuando se separan esos dos niveles, como sucede en las sociedades contemporáneas, los movimientos [...] surgen en "áreas", en redes sociales donde se negocia y configura una identidad colectiva. Las áreas de movimiento son: a) un campo en el cual se estructura mediante la negociación una identidad colectiva, en la cual orientaciones y vínculos de la acción son definidos y redefinidos dentro de unas redes de solidaridad que muestran la relación entre las personas y su vida cotidiana; b) un terreno donde la identidad se recompone y unifica: redes sociales que confieren cierta continuidad y estabilidad a las identidades de individuos y grupos en sistemas sociales donde éstas se hallan en un proceso de constante fragmentación o desestructuración. De este modo, el movimiento proporciona a individuos y grupos un punto de referencia para reconstruir identidades divididas entre distintas afiliaciones, distintos roles y tiempos de la experiencia social. (Melucci, 1994: 135-136)

En distintas ocasiones se ha señalado la necesidad de diferenciar dos momentos en el proceso de movilización social, "un modelo de funcionamiento a dos polos". La existencia de unas redes sumergidas constituidas por pequeños grupos que actúan en la vida cotidiana, en los que se implican personas que crean y experimentan modelos culturales. Estas redes se constituyen en relación con problemas específicos y en torno a ellos emergen (la paz, problemas medioambientales, discriminación de género o étnica), constituyendo un circuito de intercambios sociales. No todos los grupos en estas redes son igualmente importantes y algunos proporcionan una cierta unidad. Durante las fases de latencia o no visibilidad pública, se experimentan nuevos modelos culturales, se construyen nuevos significados y se elaboran códigos diferentes a los dominantes en la sociedad, mediante la reapropiación o resignificación, lo que favorece el cambio social. Esta fase de latencia funciona como una especie de laboratorio donde se lleva a cabo la innovación y se crean los desafíos simbólicos a los códigos dominantes en

\footnotetext{
${ }^{3}$ Repárese en la asociación que Alberto Melucci realiza entre Estado, movimiento obrero y lucha por el reconocimiento de los derechos civiles y la ciudadanía, sobre la que ya hemos insistido antes. Lo que me interesa resaltar es el hecho de que la extensión de la acción colectiva a nuevas áreas en las que aparecen procesos de construcción de identidades colectivas se puede interpretar, y yo lo haré, en términos de producción de nuevos derechos colectivos de ciudadanía. Entendida así la ciudadanía no sería algo fijo y estable sino una definición sometida a constantes interpretaciones, formulaciones (sociales y jurídicas) y transformaciones. Más como algo dinámico cuya concreción depende de concretas luchas de poder en función de momentos históricos, contextos geográficos y condiciones sociales, culturales y políticas.
} 
la sociedad. Cuando estas redes emergen se sitúan frente a una autoridad política en torno a determinadas demandas, se sitúan delante del proceso de toma de decisiones respecto a una política pública concreta.

La movilización opera como medium, es decir, indica al resto de la sociedad la relación existente entre un problema específico y la lógica dominante en el sistema. [...] Estos dos polos están recíprocamente conectados. La latencia hace posible la acción visible porque proporciona los recursos de solidaridad que necesita y produce el marco cultural dentro del cual surge la movilización. Esta última a su vez refuerza las redes sumergidas y la solidaridad entre sus miembros, crea nuevos grupos y recluta nuevos militantes atraídos por la acción pública del movimiento que pasan a formar parte de dichas redes. (Melucci, 1994: 147)

El aspecto más relevante de los movimientos sociales tanto para sus activistas como para el conjunto de la sociedad tiene que ver con su capacidad de producción simbólica. Mediante mecanismos de interacción entre los activistas se elabora la identidad de la organización o del movimiento. Esta identidad se negocia y renegocia constantemente y se interioriza mediante las prácticas sociales que tienen lugar en la vida cotidiana, la dimensión no visible de la movilización, y los actos en los que se manifiesta la protesta. La identidad colectiva es fundamental para la definición de las metas y objetivos que se persiguen, desempeñando un papel central como motivación para el mantenimiento del compromiso de los activistas. La identidad colectiva resulta también clave en la búsqueda de reconocimiento por parte de las autoridades y competidores, así como para la consecución de su aceptación pública por parte de la sociedad.

La búsqueda de reconocimiento público de intereses privados se ha tematizado históricamente a partir de la construcción de la dicotomía conceptual público versus privado. El tránsito de lo privado a lo público necesita de un momento intermedio en el que se produce la agregación de voluntades privadas mediante la constitución de redes sociales, lo que denomino privacidad compartida, y que a través de la movilización política adquiere visibilidad y notoriedad en la esfera pública. Así se han ido ampliando históricamente las esferas de la ciudadanía. Detengámonos en algunos ejemplos del proceso de construcción social de la ciudadanía en la sociedad contemporánea: feminismo, ecología, campesinos, indígenas, gays y lesbianas e inmigrantes. ${ }^{4}$

\footnotetext{
${ }^{4}$ No pretendo afirmar que estos procesos de construcción sólo se den en la sociedad contemporánea. Por el contrario, algunos de los ejemplos mencionados cuentan con una larga tradición de movilización que se remonta al siglo XIX, e incluso, es relativamente fácil encontrar precedentes anteriores.
} 
Desde finales del siglo XIX, el movimiento sufragista, primero, y el feminista, después, vienen actuando contra los mecanismos sociales y políticos que reproducen la desigualdad de género. Si nos limitamos a las sociedades de nuestro entorno europeo desarrollado, se puede afirmar que existe una igualdad formal entre hombres y mujeres, conseguida mediante los cambios introducidos en la legislación que no hace mucho tiempo legitimaba todo tipo de desigualdades. La expansión del discurso antidiscriminatorio y sobre el derecho a la igualdad ha ido penetrando en las instituciones sociales y políticas, aunque todavía se está muy lejos de una igualdad real.

En las últimas décadas se han producido profundas transformaciones como resultado del impacto de nuevos valores igualitaristas defendidos y reivindicados por el feminismo organizado y, también, por numerosos grupos de mujeres.

El movimiento feminista ha dedicado amplios esfuerzos a la construcción de una nueva identidad personal entre las mujeres, más allá de la imagen tradicional que la sociedad las adjudica. Buena parte de sus contenidos se centran en el mundo de lo personal y de la intimidad: derecho al propio cuerpo y a decidir sobre las cuestiones que las afectan, el disfrute sexual, el amor, la integridad física, etc. Muchos de los debates públicos en la actualidad giran en torno a estos temas (interrupción del embarazo, malos tratos) que constituyen una parte nuclear de la definición feminista de la identidad femenina. El reconocimiento de una nueva imagen de la mujer no heterosexual como derecho a una opción sexual diferente forma también parte de dicha conquista simbólica. El movimiento feminista ha impulsado una nueva ética, una transformación ética de la sociedad para que se tengan en cuenta los deseos y las necesidades de las mujeres, con los necesarios cambios en las costumbres, las formas de vida y los hábitos sociales. Desde los años 60, el feminismo ha venido proclamando con el lema "lo personal también es político" que sus reivindicaciones no sólo afectan a cambios legislativos, por ejemplo, también se relacionan con cambios en las prácticas sociales que atraviesan todos los ámbitos de la vida.

Entre los distintos movimientos sociales el que más ligado se encuentra desde sus orígenes a la globalización es el ecologismo. Con anterioridad, la preservación del medioambiente, como reducción tecnocrática, y la protección de la naturaleza, como valor simbólico, habían intentado frenar la voracidad incontrolada del desarrollo capitalista. En realidad, estos grupos pretendían mantener al margen del progreso, y de la colonización territorial de la explotación capitalista del entorno, aquellos lugares menos humanizados o transformados por la mano del hombre. 
Sólo recientemente se ha tomado conciencia de que el problema no está fuera sino en el interior de nuestra forma de relacionarnos con la naturaleza, en la lógica misma que impulsa el sistema de producción capitalista. La lucha contra las plantas nucleares de producción de energía y su utilización con fines militares impulsaron el despliegue de una conciencia de crisis sobre la posibilidad de acabar con el soporte físico de la vida humana. Fueron el accidente en la Central Nuclear de Three Miles Island en EE. UU. en los setenta, el establecimiento de misiles de alcance intermedio con cabezas nucleares en Europa, y el accidente en la Central Nuclear de Chernobil, los tres momentos en los que la conciencia ecologista cristalizó, proyectándose más allá de los estrictos límites locales hasta alcanzar un ámbito global.

Aunque la identidad del movimiento ecologista puede que no sea tan clara y fuerte como la del movimiento feminista, no hay duda de que las movilizaciones recientes han extendido sus valores hasta transformar nuestra concepción de los problemas y de sus soluciones. La crítica al modelo de desarrollo económico tiene en su transfondo una fuerte carga moral: la obligación moral con el $75 \%$ de la humanidad que no disfruta de las promesas de la sociedad de consumo, pero sufre sus consecuencias negativas (miseria, injusticias de todo tipo, exclusión social). Implícito en el discurso ecologista encontramos también una nueva antropología, una redefinición de nuestras señas de identidad personal y colectiva, una nueva forma de relacionarse con nuestro entorno y, por lo tanto, de estar en sociedad.

El discurso del ecologismo ha ido evolucionando desde los problemas locales a los globales, ya que las manifestaciones locales de las agresiones al medioambiente responden a una lógica que funciona a nivel global. No es este o aquel problema lo que hay que denunciar (el síntoma que denuncia la enfermedad) sino el modelo de desarrollo capitalista (la enfermedad) que los produce. Este modelo descansa sobre dos pilares: la idea de progreso que necesita del crecimiento (progreso económico que conduciría a un progreso social que llevaría a un progreso humano), y la idea de que es a través del consumo de todo tipo de bienes como llegaremos a satisfacer nuestras necesidades presentes y futuras. Como han señalado Morin, Galbraith o Baudrillard no es a través del crecimiento como solucionaremos nuestros problemas de progreso, y de ello se ha hecho eco el movimiento ecologista cuando propugna un cambio del modelo de desarrollo centrado en el crecimiento, y de las actitudes y prácticas sociales que implica. 
La ecología ha dejado de ser antropo y eurocéntrica para convertirse en global, incorporando en sus propuestas la necesidad de contemplar las relaciones norte-sur y no centrarse sólo en los países desarrollados. ${ }^{5}$

Más allá de la diversidad de manifestaciones de la movilización feminista, uno de sus logros fundamentales ha sido la construcción de una identidad para la mujer que ha roto con su dependencia de la cultura masculina. Identidad que implica una progresiva transformación de las costumbres, de las formas de vida, de los hábitos y de las formas de pensar dominantes. Uno de los temas prioritarios del feminismo es la redefinición de los valores dominantes, uno de cuyos pilares es el sistema educativo del que se pregona su reforma en el camino de la coeducación, que implicaría una nueva ética que conduciría a una nueva sociedad. De su necesidad nos habla no sólo el movimiento feminista sino también sus ramificaciones en lo que podemos denominar el movimiento amplio de mujeres. Este movimiento ha sido capaz de aunar en el interior de sus numerosísimos grupos un espacio de refugio, de vida, relación y sentido para el conjunto de las mujeres. El reto consiste en convertirlos en derechos de ciudadanía en las prácticas tanto públicas como privadas.

Antes de pasar a otros tipos de movilización recuperemos las diversas relaciones entre lo público y lo privado que hemos encontrado en una investigación realizada en España recientemente. ${ }^{6}$

\footnotetext{
${ }_{5}^{5}$ Desde la política ecológica y la teoría política se han formulado recientemente conceptos como el de ciudadanía planetaria (Steward, 1991) o el de ciudadanía ecológica, sobre el que se ha producido recientemente un extenso debate (Barry, 1996 y 1999; Barry y Proops, 2000; Christoff, 1996; Dobson, 2000 y 2001). Como ha señalado Ángel Valencia: "la tesis de Dobson es que hay 'una arquitectura conceptual de la ciudadanía' que se basa en tres grandes elementos: la ciudadanía entendida como reclamación de derechos; la esfera pública como el lugar idóneo para la práctica de la ciudadanía; y la ciudadanía como una relación recíproca entre el ciudadano y las instituciones políticas constituidas. La ciudadanía ecológica constituye una versión paralela - una versión subordinada - a esta concepción tradicional, dominante, de la ciudadanía. Tiene la misma estructura, pero los puntos de referencia son radicalmente opuestos. En esta versión alternativa, el ciudadano tiene responsabilidades además de derechos, y no hay ninguna relación recíproca entre los dos. Asimismo, la esfera privada se convierte en un lugar donde se hace ciudadanía, no un sitio de donde hay que escapar para practicarla. Y por último, la conexión entre la ciudadanía y un territorio político específico se deshace a favor de una concepción 'desterritorializada' de la ciudadanía. Mi propósito no es el de descartar el concepto tradicional de la ciudadanía - de suma importancia en muchos contextos. Pero sí quiero resaltar que en un mundo globalizado y azotado por problemas globales hace falta una ciudadanía que esté a la altura de los tiempos" (Valencia, 2004: 102). Lo que está reclamando Valencia con este cambio de concepción es, entre otras cosas, que la dimensión privada, las prácticas que los actores sociales llevan a cabo en la vida privada, no quede excluida de la ciudadanía ecológica. En ella lo público y lo privado (espacio público y espacio privado) quedarían vinculados por una privacidad compartida constituida por prácticas sociales que, al mismo tiempo, ponen en funcionamiento derechos y se responsabilizan de los efectos de dichas prácticas.

${ }^{6}$ Incorporamos algunos testimonios de activistas ecologistas tomados de la investigación "Socialidad, participación y movilización en la sociedad española: Nuevas formas de construcción social de la identidad y el sentido".
} 
Desde algunas interpretaciones sociológicas se ha intentado comprender la participación política y la intervención en acciones colectivas como una superación/rechazo de las tendencias a la privatización de la vida, tendencias que serían dominantes en las sociedades modernas. El privatismo civil (profesional y familiar) atraería los esfuerzos del hombre y mujer modernos (Habermas), dejando en manos del estado la parte necesaria de "solidaridad colectiva". El abandono de la esfera pública, por parte del ciudadano común, vendría impuesto por la preferencia a buscar su autorrealización en el ámbito de la esfera privada y de la intimidad. Las tendencias a la privatización de la vida presentes en buena parte de las sociedades industriales avanzadas vendrían a explicar la creciente desafección de los ciudadanos por la discusión y participación en los asuntos públicos/políticos. Aunque reconozcamos la certeza de esta explicación para comprender algunos cambios históricos que afectan a determinadas generaciones de diversas sociedades, es insuficiente para dar cuenta de las complejas relaciones que los actores atribuyen a la dicotomía público/privado y político/personal. Veamos algunas de estas dimensiones.

En el grupo realizado con ecologistas valencianos, los participantes se refieren a cómo la movilización transforma las motivaciones originarias, cargadas de romanticismo, en motivaciones heroicas que requieren de un compromiso personal profundo, es decir, se convierte en parte constitutiva de la relación que los activistas establecen entre su mundo privado e íntimo con la esfera pública:

Adena/Greenpeace: Sí, conforme estás aquí aumenta tu visión global, te das cuenta de que todo está interrelacionado, además de medio ambiente estamos sacando temas de dignidad humana, de calidad de vida, estamos contemplando en realidad todo y creo que las motivaciones sí que cambian, porque cuando entramos yo creo que entramos todos de forma un poco romántica. Y cuando empiezas a llevar días y días y años... entonces te das cuenta de que...

Acción Ecologista Agró C1: Se convierte en un compromiso ya...

Adena/Greenpeace: Sí, se convierte en un compromiso y a lo mejor es un poco más duro de lo que tú te esperabas en un principio, un poco más duro, un poco más difícil, desaparece la parte romántica y te das cuenta de que hay que pelear mucho a diario... [...] Puedes entrar de una forma romántica y se convierte en un compromiso personal, un compromiso muy profundo.

La búsqueda de la realización personal, la autorrealización a nivel privado, conduce al compromiso público, intentando aportar un grano de arena más 
a la transformación colectiva de la sociedad; en palabras de una activista ecologista "la coherencia personal exige la acción pública":

Si yo creo que está dicho, o sea, si llevamos tantos años en un sitio y en otro es porque sí que creemos que, no sé si eso que has dicho de la existencia o no sé qué de la vida ¿no? Pues de alguna forma sí que crees que no estas aquí, por lo menos yo sí me siento mejor conmigo misma haciendo algo por... No por cambiar porque sabemos que a pesar de lo que hacemos, no cambia mucho, pero sí por intentarlo, sí por coherencia con una misma, por coherencia con una misma y no con nadie más, sino conmigo misma.

Para algunos activistas, sin embargo, sí existe una separación clara entre vida privada y activismo, difíciles de compatibilizar. Un activista ecologista se refiere a la recuperación de su vida privada ahora, en contraste con lo que sucedía anteriormente, cuando la movilización absorbía todos los esfuerzos y todas las actividades:

Y claro, ahora tenemos nuestra vida, yo creo que eso sí hemos recuperado. Es una parte de vida personal: que te gusta ir al cine, te gusta leer, te gusta salir por ahí, te gusta andar con otra gente, que no es sólo la de tu grupo, si no esto sería..., esto sí que sería aburridísimo.

La implicación profunda en la militancia puede llegar a modificar completamente tu vida personal, mediante la transformación de ciertos estilos de vida, de pautas de comportamiento. La acción en la esfera pública conduce a una transformación de la esfera privada. Existen ejemplos ya conocidos como la participación en grupos religiosos cerrados o en organizaciones clandestinas, pero también hay numerosísimos ejemplos entre feministas o en el ámbito de la movilización ecologista:

Entrevistador: El hecho de participar en el movimiento ecologista cos ha cambiado vuestra forma de vivir?, ¿de vivir la vida?

Acción Ecologista Agró V1: Lógico... si estás todos los días ahí y un montón de años, pues te... te cambia... si eres consecuente lógicamente.

Acción Ecologista Agró V2: Te va cambiando un poco la conciencia de tu forma de vida y de las cosas que tú puedes hacer, a parte de la lucha diaria, en tu vida personal... Adena/Greenpeace: Totalmente, a mí me ha cambiado hasta la dieta, yo ahora soy prácticamente vegetariano... sí, sí... yo creo que te cambia totalmente porque el conocimiento te va aportando una serie de cosas, aumenta tu grado de compromiso y ese grado de compromiso acaba por afectar a toda tu vida. 
Acción Ecologista Agró C1: Implica tiempo e implica muchas cosas...

Adena/Greenpeace: Claro... e implica... cambia totalmente, si te implicas de verdad y, para seguir, tienes que implicarte de verdad, al final cambia totalmente tu concepción de la vida... de todo.

La dicotomía público/privado tiene su correlato en otra dicotomía que funciona como equivalencia entre político y personal (no político). Así, mientras lo público es político no privatizable, ni susceptible de apropiación personal (la infracción de esta regla llevaría a la corrupción o apropiación indebida), lo personal es apolítico, no susceptible de publificación colectiva, esfera resguardada de la intromisión del público y de la política (escrutinio público). Lo privado es lo que está fuera del alcance del escrutinio público y de los intentos de visibilización por parte del público: lo opaco de la vida del yo frente a "los otros". Pero es visible para el yo en términos de conciencia de tu forma de vida y de cosas que tú puedes hacer (coherencia personal) y de lucha diaria en tu vida personal (transformación personal).

El MST (Movimiento brasileño de los trabajadores sin-tierra) aparece en 1979 como resultado del proceso histórico de resistencia del campesinado brasileño frente a la expropiación, la expulsión y el trabajo asalariado de centenares de miles de pequeños agricultores y campesinos sin tierra. Su momento fundacional puede fecharse el 7 de septiembre de 1979 cuando se lleva a cabo la ocupación de la finca Macali en Ronda Alta (Rio Grande del Sur). Esta fue una acción a partir de la que se produce la gestación del MST (Mançano Fernandes, 2000: 50) que utiliza como forma de lucha la ocupación de tierras y el asentamiento de agricultores y campesinos sin tierra en los espacios ocupados. Sin embargo, los objetivos más amplios tratarían de alcanzar una reforma agraria y una democratización de la propiedad de la tierra en un país de más de 8,000.000 de km2 con centenares de miles de trabajadores rurales sin acceso a la tierra y con el mayor grado de concentración de la tierra del planeta.

Junto al MST existen en Brasil más de 15 movimientos sociales que luchan por la tierra. El mismo tipo de movimiento encontramos en el noroeste de Argentina, Ecuador, Bolivia, Colombia, México, Sudáfrica o Zimbabwe. Para muchos millones de personas la única forma de insertarse en la globalización es luchar por la supervivencia trabajando un pedazo de tierra, transformando el acceso a la propiedad o uso de la tierra en un derecho democrático. La posibilidad de disponer de tierra para trabajarla y con ello garantizarse su subsistencia se ha convertido en una de las demandas más acuciantes de millones de seres humanos a lo largo del planeta, demanda que sin su cumplimiento reduce a estas personas a una situación de pre-ciudadanía. 
A medio camino entre la ciudadanía inclusiva y la diversidad cultural creativa, encontramos al Movimiento Zapatista. Su genealogía es bien conocida, sobre todo desde el 1 de enero de 1994 cuando su acción reivindicativa salta de la Sierra La Candona a la escena internacional. Su objetivo se centra en reclamar el derecho a la tierra y al desarrollo de los pueblos indígenas, y el respeto y salvaguarda de culturas minoritarias debilitadas por la presión de otras culturas dominantes.

El Movimiento Zapatista ha sido durante varios años el espejo en el que se han visto reflejados buena parte de los pueblos indígenas, sobre todo, de México y América Latina. Su relativo éxito sustantivo no hace sino agrandar las virtudes del liderazgo y el acierto de la línea estratégica de movilización adoptada.

Además de la necesidad de reservar un espacio a la movilización indígena, que ha alcanzado distinto grado de éxito en el proceso de redefinición y lucha por el reconocimiento de los derechos de ciudadanía, en países como Colombia, Guatemala, Bolivia o Ecuador, merece la pena prestar atención a todas aquellas formas de defensa de identidades colectivas que se producen en Estados-nación ya constituidos, que suelen ir acompañados de formas culturales y lingüísticas propias. Prácticamente no existe país alguno en el que no se produzcan manifestaciones de esta reivindicación de carácter etnolingǘrstico o nacionalista que nos habla de la diversidad cultural que ha sobrevivido a los impulsos homogeneizadores del Estado-nación moderno (vascos, catalanes, gallegos, bretones, corsos, occitanos, galeses, escoceses, quebequeses o sardos).

El movimiento gay alcanzó su visibilidad pública la noche del 27 al 28 de junio de 1969 en Nueva York, cuando un grupo de "drag queens", chicos de alterne, hombres que ejercían la prostitución y gente de la calle se enfrentaron a la policía en el Greenwich Village después de una serie de redadas en bares gays, entre ellos uno llamado Stonewall. Con posterioridad a aquellos acontecimientos, el movimiento gay experimentó una rápida politización cuando nuevos militantes procedentes de sectores estudiantiles y de la Nueva Izquierda introdujeron sus debates y sus concepciones políticas en el ámbito del género y de la sexualidad (Adam, 1987: 76).

Si por algo me parece interesante situar aquí al movimiento gay es porque lo considero paradigmático de cómo se construye socialmente una identidad sobre la condición de la norma no heterosexual frente a una sociedad que o bien la rechaza o, en unos pocos casos, es permisiva hacia dicha norma.

La condición de homosexual, transexual o cualquier otra orientación sexual que se aleje de la pauta heterosexual dominante implica un proceso de socialización, de aprendizaje, de asunción o de toma de conciencia que 
precisa la creación de una estructura de plausibilidad en la que los individuos que abrazan dicha orientación o condición puedan encontrar apoyo a sus creencias y prácticas, y a partir de ellas construir una identidad personal y colectiva desprovista de los elementos negativos y estigmatizantes que les atribuyen otros colectivos sociales. La lucha por el reconocimiento de la igualdad de gays, lesbianas y transexuales, y por "su normalización social", pasa por reformular el ámbito de aplicación de los derechos de ciudadanía, para extenderlos a quienes no sólo son partidarios de las prácticas heterosexuales, las lleven a cabo o no. ${ }^{7}$

Ralf Dahrendorf (1990) afirma que el conflicto social moderno se ha desplazado del conflicto que desgarraba a las sociedades industriales en dos clases antagónicas hacia el problema de la inclusión social. La cuestión social hoy tendría que ver con los mecanismos que en las sociedades avanzadas producen de manera sistémica exclusión social. De la mano de la necesidad de dar respuesta a estos colectivos cada vez más numerosos se ha ido consolidando lo que en otro lugar he llamado el movimiento de solidaridad.

Las organizaciones y grupos que configuran estas nuevas formas emergentes de movimientos sociales actúan en el ámbito de la solidaridad con los sectores menos favorecidos o marginados de las sociedades occidentales, así como con colectivos que se han visto impulsados a emigrar buscando mejorar su condición económica o su seguridad. Asociaciones antirracistas o de apoyo y colaboración al desarrollo muestran señales de relevantes diferencias con los movimientos sociales más consolidados.

La significación que estas organizaciones han adquirido en los últimos años es de tal magnitud que ya ha comenzado a influir en las formas de ser y actuar de los movimientos sociales tradicionales. De hecho muchos grupos ecologistas, pacifistas o ligados al mundo del trabajo inician su actividad pública eligiendo o imitando las formas tradicionales y las estructuras de los movimientos por la solidaridad. Este nuevo escenario nos permite reflexionar sobre las relaciones entre contexto histórico y movimientos sociales y, eventualmente, plantear la cuestión de si no estaremos asistiendo a una nueva fase, o al menos, a nuevas formas, de acción colectiva. Aunque todavía es pronto para así afirmarlo, pensamos que esta hipótesis ha de rastrearse y analizarse seriamente para ver hasta qué punto esta amalgama de elementos nuevos y antiguos pudiera estar configurando una realidad novedosa (Ibarra y Tejerina, 1998: 10 y ss.).

\footnotetext{
${ }^{7}$ De las dificultades y resistencias sociales frente a los intentos de equiparar en derechos a todos los ciudadanos valga de ejemplo el reciente intento de reconocer igualdad de derechos para contraer matrimonio civil a cualquier persona independientemente de sus preferencias u orientación sexual experimentado en España durante la primavera de 2005.
} 
Estos movimientos no sólo testimonian sino que en muchos casos lideran la movilización social, ya sea porque han alcanzado mayor presencia en la esfera pública o porque los otros movimientos imitan, de forma creciente, en sus formas reivindicativas e identitarias, a los movimientos sociales por la solidaridad. La movilización en favor de la regulación de inmigrantes y del reconocimiento de la diversidad cultural característica de las modernas sociedades multiculturales constituiría otro caso de los procesos de ampliación del elenco tradicional de los derechos de ciudadanía

\section{Consideraciones finales}

La lógica social que orienta la acción de los movimientos sociales contemporáneos guarda relación con los procesos de construcción y ampliación de la ciudadanía. Los contenidos de la ciudadanía, tal y como los había definido T.H. Marshall, se habían extendido desde el siglo XVIII, adquiriendo nuevas redefiniciones de la mano de los conflictos de clase característicos de la sociedad industrial. Estos derechos de ciudadanía, construidos a partir de las características de los protagonistas que actuaban en la esfera pública, dejaban fuera o en los márgenes de su aplicación a amplios sectores sociales. Los logros de la ciudadanía en la sociedad industrial están directamente relacionados con las movilizaciones sociales que ocupando el espacio público llevaron a cabo demandas políticas frente a las autoridades.

La crisis de la doble centralidad de la sociedad moderna, social y política, de clase y de interés (del juego entre intereses y del reparto de los intereses en juego) posibilita la emergencia en la esfera pública de nuevas definiciones de la ciudadanía que la lógica social dominante de la sociedad moderna había excluido. La crisis aparece como una crisis multipolar, compleja. Pero lo característico de esta crisis es que, por un lado, se manifiesta públicamente pero no se genera en el espacio público y, por otro lado, se articula en un espacio intersticial no institucionalizado, en las costuras entre la vida cotidiana y los resquicios de la política no institucional. La cuestión que plantea la movilización social a partir de la década de los años 70 es de distribución sí, pero antes que de redistribución plantea un debate previo sobre el reconocimiento, sobre la legitimidad del "nosotros" antes que de la legitimidad de "nuestras demandas".

Los procesos de construcción del "nosotros" remiten a los mecanismos de interacción que actúan, en parte, en una esfera de semiprivacidad o de privacidad compartida y, en parte, en la esfera pública cuando alcanzan un grado mayor de visibilidad social. Visibilidad e inteligibilidad que no serían posibles al margen del funcionamiento en redes sociales y de interacción donde se experimentan y reformulan identidad e intereses. Estos procesos 
tienen un alto grado de reflexividad, mediante la que el autoconocimiento se transforma en conocimiento social. Quizás una de las condiciones de posibilidad de la sociedad tecnoinformacional sea el predominio que los componentes simbólicos ejercen sobre los procesos de reproducción material: la política y la tecnología.

Los movimientos sociales contemporáneos señalan un doble proceso de crisis: por implosión de la ciudadanía y por explosión de la política como espacio autónomo producido en el proceso de diferenciación social. En relación con la implosión de la ciudadanía he señalado varios ejemplos de reformulación de la concepción tradicional de la ciudadanía mediante la movilización social: feministas, ecologistas, campesinos, indígenas, etnonacionalistas, solidaridad, gays, lesbianas y transexuales. Es posible que, en esta misma línea de reflexión, la acción colectiva a favor de una globalización alternativa represente la emergencia de una ciudadanía global o planetaria. En relación con la explosión de la política como un espacio de diferenciación social asistimos a procesos de desdiferenciación social que nos obligan a repensar la distinción tradicional y las relaciones entre las categorías de lo personal y lo político, la esfera privada y la esfera pública. No se trata ya de constatar la aparición de un espacio de política no institucional, que ya se ha institucionalizado ( 20 no son instituciones sociales los movimientos sociales?), sino de unos intereses e identidad personales que al ser producidos y compartidos socialmente se transforman en públicos sin dejar de tener necesariamente que estar presentes en un espacio personal, pero también, y sobre todo, de privacidad compartida.

Algunas de las formas de la ciudadanía que se ensayan en los movimientos sociales contemporáneos no se orientan a la política como ámbito privilegiado del reconocimiento y mundo de la producción social, sino hacia una esfera post-privatista o pre-política, como ámbito privilegiado de transformación personal (ser y no representar, conocer y no reconocer) y del orden simbólico de la relación cara a cara. Así, junto al sentido tradicional de la separación entre lo público-político y lo privado-personal aparecen nuevos sentidos: a) cuando se afirma que lo público no existe sin la participación de lo privado, luego la acción política implica necesariamente "mi" contribución personal; b) cuando se afirma que lo político es profundamente personal, puesto que la búsqueda de coherencia personal exige la acción pública (como toma de conciencia de tu forma de vida frente a un problema general); c) cuando se afirma que lo político es lo que afecta a lo íntimo y, por lo tanto, sólo la transformación de lo íntimo es auténticamente político (lo que cada uno puede hacer en su vida personal como parte de una lucha diaria). Estos sentidos inducen a la participación en la movilización social, 
a la emergencia de nuevos sentidos de la ciudadanía en las condiciones de individualización y producción de la identidad colectiva características de la sociedad contemporánea, en una constante redefinición de los límites de la esfera pública.

\section{Referencias Bibliográficas}

Adam, Barry D. (1987), The Rise of a Gay and Lesbian Movement. Boston: Twayne Publishers.

Alonso, Luis Enrique (2004), “¿Redistribución o reconocimiento? Un debate sociológicamente no siempre bien planteado”. V Encuentros de Teoría Sociológica. Valencia. Barry, John (1996), "Sustainability, Political Judgement and Citizenship: Connecting Green Politics and Democracy", in Brian Doherty; Marius de Geus (orgs.), Democracy and Green Political Thought: Sustainability, Rights and Citizenship. London: Routledge.

Barry, John (1999), Retbinking Green Politics. Nature, Virtue and Progress. London: Sage.

Barry, John; Proops, John (2000), Citizenship, Sustainability and Environmental Research. Q Methodology and Local Exchange Trading Systems. Cheltenham: Edward Elgar. Bottomore, Tom (1998), Ciudadanía y clase social, cuarenta años después, in T. H. Marshall; Tom Bottomore, Ciudadanía y clase social. Madrid: Alianza, 85-142.

Brubaker, William Rogers (1992), Citizenship and Nationbood in France and Germany. Cambridge, Mass.: Harvard University Press.

Castells, Manuel (1998), La era de la información: Economía, Sociedad y Cultura. El fin del milenio. Volumen III, Madrid: Alianza.

Christoff, P. (1996), "Ecological Citizens and Ecologically Guided Democracy”, in Brian Doherty; Marius de Geus (orgs.), Democracy and Green Political Thought: Sustainability, Rights and Citizenship. London: Routledge.

Dahrendorf, Ralf (1990), El conflicto social moderno. Ensayo sobre la política de la libertad. Madrid: Mondadori.

Dobson, Andrew (2000), Green Political Thought. London: Routledge.

Dobson, Andrew (2001), "Cambios en la ciudadanía”, in R. Maíz (org.), Europa Mundi. Construcción de Europa, democracia y globalización. Santiago de Compostela: Universidade de Santiago de Compostela. Vol 2.

Dubet, François (2003), "Mutaciones cruzadas: La ciudadanía y la escuela”, in Jorge Benedicto; María Luz Morán (orgs.), Aprendiendo a ser ciudadanos. Experiencias sociales y construcción de la ciudadanía entre los jóvenes. Madrid: Injuve, 219-234.

Eder, Klaus (1998), "La institucionalización de la acción colectiva. ¿Hacia una nueva problemática teórica en el análisis de los movimientos sociales”, in Pedro Ibarra; Benjamín Tejerina (orgs.), Los movimientos sociales. Transformaciones políticas y cambio cultural. Madrid: Trotta, 337-360. 
Ferre, Myra M. (1994), “El contexto político de la racionalidad: las teorías de la elección racional y la movilización de recursos”, in E. Laraña; J. Gusfield, Los nuevos movimientos sociales. De la ideología a la identidad. Madrid: CIS, 151-182.

Ibarra, Pedro; Tejerina, Benjamín (orgs.) (1998), Los movimientos sociales. Transformaciones políticas y cambio cultural. Madrid: Trotta.

Inglehart, Ronald (1991), El cambio cultural en las sociedades industriales avanzadas. Madrid: CIS-Siglo XXI.

Mançano Fernandez, Bernardo (2000), A formação do MST no Brasil. Petrópolis: Vozes.

Marshall, Alfred (1925), “The Future of the Working Classes”, in A. C. Pigou (org.), Memorials of Alfred Marshall. London: Macmillan.

Marshall, Thomas Humphrey (1998), Ciudadania y clase social, in T. H. Marshall; Tom Bottomore (1998), Ciudadanía y clase social. Madrid: Alianza, 15-82.

Martínez de Albeniz, Iñaki (2003), La poética de la política. Usos de la política en el País Vasco. Bilbao: Servicio Editorial de la Universidad del País Vasco.

Melucci, Alberto (1989), Nomads of the Present: Social Movements and Individual Needs in Contemporary Society. London: Hutchinson Radius.

Melucci, Alberto (1994), “¿Qué hay de nuevo en los 'nuevos movimientos sociales’?”, in Enrique Laraña; Joseph Gusfield (orgs.), Los nuevos movimientos sociales: De la ideología a la identidad. Madrid: CIS, 119-149.

Melucci, Alberto (1995), "The Process of Collective Identity", in Hank Johnston; Bert Klandermans (orgs.), Social Movements and Culture. London: UCL Press, 41-63.

Melucci, Alberto (1996), Challenging Codes. Collective Action in the Information Age. Cambridge: Cambridge University Press.

Offe, Claus (1988), Partidos politicos y nuevos movimientos sociales. Madrid: Sistema.

Olson, Mancur (1965), The Logic of Collective Action: Public Goods and the Theory of Groups. Cambridge, Mass.: Harvard University Press.

Pizzorno, Alessandro (1994), "Identidad e Interés”, Zona Abierta, 69, 136-143.

Steward, F. (1991), "Citizens of the Planet Earth”, in Geoff Andrews (org.), Citizenship. London: Lawrence and Wishart.

Tejerina, Benjamín (1998), "Los movimientos sociales y la acción colectiva. De la producción simbólica al cambio de valores”, in Pedro Ibarra; Benjamín Tejerina (orgs.), Los movimientos sociales. Transformaciones politicas y cambio cultural. Madrid: Trotta, 111-138.

Tejerina, Benjamín et al. (1995), Sociedad civil, protesta y movimientos sociales en el País Vasco. Los límites de la teoría de la movilización de recursos. Vitoria-Gasteiz: Servicio Central de Publicaciones del Gobierno Vasco.

Tejerina, Benjamín; Martínez de Albéniz, Iñaki (1999), "Movimientos sociales y globalización en Euskal Herria”, in J. Beriain; R. Fernández Ubieta (orgs.), La cuestión vasca. Claves de un conflicto cultural y político. Barcelona: Proyecto A, 244-256. 
Touraine, Alain (1978), La voix et le regard. Paris: Editions du Seuil.

Valencia, Ángel (2004), "Certezas y dilemas de la ciudadanía ecológica dentro de una política global”, in Jorge Riechmann (org.), Ética ecológica. Propuestas para una reorientación. Montevideo: Nordan-Comunidad, 83-107. 